\title{
Las políticas de convivencia escolar colombianas en el contexto de América Latina
}

\author{
Francisco Javier Benítez Moreno ${ }^{1}$ \\ I.E.M. Ciudadela Educativa de Pasto, Colombia
}

Recepción: 23/04/2020

Evaluación: $30 / 04 / 2020$

Aprobación: 10/05/2020

Artículo de Investigación-Reflexión

DOI: https://doi.org/10.22267/rhec.202424.72

\section{Resumen}

En las instituciones educativas colombianas se gestan fenómenos como agresión, intolerancia, venta y consumo de drogas. Estas situaciones inciden en el disfrute de los derechos de los menores por lo que el Estado se encuentra en la obligación de desplegar acciones conducentes a minimizar los factores de riesgo. El propósito de este trabajo fue analizar las políticas de convivencia escolar colombianas en el contexto de América Latina, para develar los enfoques y aspectos importantes en la mejora de las relaciones interpersonales. La metodología fue revisión y análisis documental de fuentes, como leyes, decretos, sentencias de la corte constitucional, guías ministeriales, tesis y artículos que condujeran a la comprensión de tres aspectos: la fundamentación jurídica, las alternativas implantadas y las dimensiones no consideradas. Según los enfoques de comprensión para Latinoamérica, se encuentra que Colombia se orienta por la visión democrática, lo que significa formar para el ejercicio de los derechos humanos desde los valores de la república, tales como la libertad, la justicia y dignidad; en función de ello se desarrolla la pedagogía más allá de la interacción docente-estudiante que permitiera establecer un sistema articulado con entidades externas de tipo administrativo y de salud. Los resultados de la política resaltan la necesidad de tratar el papel de las

1 Profesor orientador en la Institución Educativa Municipal Ciudadela Educativa de Pasto, Colombia. Estudiante del programa de doctorado en Ciencias de la Educación, Rudecolombia. Grupo de investigación: Psicología y Salud. Linea de investigación: salud mental, suicidio y violencia. Correo electrónico: Javierbenitez07@gmail.com. https://orcid.org/0000-0003-0686-8372 
familias en la aparición de la violencia, toda vez que su influencia en la gestión del conflicto escolar es evidente, así como, también, fortalecer las capacidades al interior de las instituciones educativas, en especial en los niveles de atención y seguimiento a la convivencia.

Palabras clave: política educacional; convivencia escolar; educación para la paz.

\title{
Colombian school coexistence policies in the context of Latin America
}

\begin{abstract}
In Colombian educational institutions, phenomena such as aggression, intolerance, sale and consumption of drugs are being developed. These situations affect the enjoyment of the rights of minors, so the state is obliged to implement actions to minimize such risk factors. The purpose of this study was to analyze Colombian school coexistence policies in the context of Latin America in order to reveal the approaches and important aspects in the improvement of interpersonal relationships. The methodology was to review and analyze documents from sources such as laws, decrees, court rulings, ministerial guides, theses and articles that lead to the understanding of three aspects: the legal basis, the alternatives implemented and the dimensions not considered. According to the approaches of understanding around and within Latin America, it is found that Colombia is guided by the democratic vision which means to form for the exercise of the human rights from the values of the republic such as freedom, justice and dignity; according to this process the interaction between the student and the teacher goes further the actual classroom, the process enables a relationship with external administrative entities and the health organizations. The results of the policy highlight the need to address the role of families in the emergence of violence, since their influence on the management of the school conflict is evident, as well as to strengthen the capacities within educational institutions, especially at the levels of attention and follow-up to coexistence.
\end{abstract}

Keywords: educational policies; school coexistence; peace education. 


\section{Políticas de convivência escolar colombiana no contexto da América Latina}

\section{Resumo}

Nas instituições educacionais colombianas, fenômenos como agressão, intolerância, venda e consumo de drogas se desenvolvem. Essas situações afetam o gozo dos direitos dos menores para os quais o Estado é obrigado a desenvolver ações que favoreçam a minimização dos fatores de risco. $\mathrm{O}$ objetivo deste trabalho foi analisar as políticas de convivência escolar colombiana no contexto da América Latina para revelar as abordagens e aspectos importantes na melhoria das relações interpessoais. A metodologia consistiuna revisão e análise documentalde fontes comoleis, decretos, decisões judiciais, guias ministeriais, teses e artigos que conduzem à compreensão de três aspectos: o fundamento jurídico, as alternativas implementadas e as dimensões não consideradas. De acordo com as abordagens de entendimento para a América Latina, verifica-se que a Colômbia está orientada pela visão democrática, o que significa formar para o exercício dos direitos humanos a partir dos valores da república como liberdade, justiça e dignidade, com base neste desenvolvimento. Pedagógico para além da interação professoraluno que permite estabelecer um sistema articulado com entidades externas administrativas e de saúde. Os resultados da política destacam a necessidade de abordar o papel das famílias no surgimento da violência, visto que sua influência na gestão do conflito escolar é evidente, bem como de fortalecer as capacidades dentro das instituições educacionais, especialmente nos níveis de atenção e acompanhamento da convivência.

Palavras-chave: política educacional; vida escolar; educação para a paz.

\section{Introducción}

Analizar las políticas públicas de convivencia escolar colombianas requiere partir de precisiones conceptuales. Por política pública se entiende el conjunto de decisiones, objetivos y acciones que lleva a cabo un gobierno para solucionar los problemas que, en un momento determinado, los 
ciudadanos y sus dirigentes consideran prioritarios. ${ }^{2}$ La forma como se define el fenómeno determina la formulación y adopción de alternativas. Desde este punto de vista, la política educativa corresponde a los modos como se diseñan estrategias para garantizar el derecho a la educación consignado en la Constitución de 1991. ${ }^{3}$ Más específicamente, las políticas de convivencia escolar se entienden como las acciones, decisiones y omisiones que se generan en el espacio público para responder a los problemas sociales que supone la coexistencia entre los actores de la comunidad educativa. ${ }^{4}$

Ahora bien, ¿qué entiende el gobierno colombiano por convivencia escolar? El Ministerio de Educación Nacional (MEN) la define como "la acción de vivir en compañía de otras personas en el contexto escolar de manera pacífica y armónica. Se refiere al conjunto de relaciones que ocurren entre las personas que hacen parte de la comunidad educativa". ${ }^{5}$ El concepto se centra en la relación interpersonal y, de acuerdo con Fierro y Carvajal, ${ }^{6}$ sería reduccionista si no considera la gestión en todos sus ámbitos: el trabajo y la participación en el aula, así como, también, la organización escolar.

La convivencia escolar, en su acepción más amplia, la darían tres ámbitos: 1) el pedagógico y curricular, 2) el organizativo/administrativo y 3) el socio-comunitario. ${ }^{7}$ El primero remite a las prácticas docentes en su manejo de aspectos didácticos relativos a la planeación, desarrollo curricular y prácticas de enseñanza orientadas a la construcción de aprendizajes. El segundo tiene que ver con las prácticas directivas involucradas en los procesos de gestión institucional para la construcción de un proyecto educativo escolar. El tercero se orienta a establecer vínculos con los padres de familia, la comunidad externa en la que se sitúa la escuela y, también,

2 Manuel Tamayo, "El análisis de las políticas públicas”, en La nueva administración pública, comps. Rafael Bañón y Ernesto Carrillo (Madrid: Alianza Editorial, 1997).

3 Rosa Pulido Valero et al., "La mediación escolar en la comunidad de Madrid: Análisis del impacto de la formación en el profesorado y alumnado en el IES Las Américas de Parla”, Revista de Mediación. Año 3, No. 6 (2010).

${ }^{4}$ Figueredo Ramírez, Carlos Arturo et al., "Las políticas públicas educativas en Colombia y su pertinencia en los planes de desarrollo". (Tesis de maestría, Universidad San Buenaventura, 2016).

5 Ministerio de Educación Nacional. Guía No. 49. Guías Pedagógicas para la Convivencia Escolar. Ley 1620 de 2013 - Decreto 1965 de 2013 (Bogotá: Ministerio de Educación Nacional, 2014), p. 25

${ }^{6}$ Cecilia Fierro-Evans y Patricia Carbajal-Padilla, "Convivencia escolar: una revisión del concepto", Psicoperspectivas. Vol. 18. No. 1 (2019).

7 Fierro-Evans y Carbajal-Padilla, "Convivencia escolar: una revisión del concepto". 
otras dependencias e instancias de apoyo. A su vez, cada uno de los ámbitos se desarrolla a partir de tres dimensiones: la inclusión, la equidad y la participación y manejo de conflictos. ${ }^{8}$

Los ámbitos y las dimensiones de la vida compartida expuestos en Carvajal y Fierro $^{9}$ integran los enfoques de la convivencia escolar: 1) como estudio del clima, la violencia o su prevención; 2) educación para la ciudadanía; 3) la paz; 4) los derechos humanos; 5) el desarrollo moral y la formación en valores. El Estado de Colombia, desde la definición que da el MEN, ${ }^{10}$ le apuesta al enfoque de educación para la paz y los derechos humanos en la medida en que posibilita reconocer la forma como construye ciudadanos respetuosos de la ley, capaces de resolver sus conflictos de manera adecuada, sin recurrir a la violencia. ${ }^{11}$

En este trabajo, se detallan las políticas de convivencia escolar colombianas en el contexto de Latinoamérica, para reflexionar según las cosmovisiones que lo inspiran y los aspectos importantes en la mejora de las relaciones interpersonales. El estudio se realiza a partir de la fundamentación jurídica, las alternativas implantadas y los aspectos no considerados en la atención a la problemática.

\section{Metodología}

La metodología empleada fue revisión y análisis documental, que consiste en extraer el contenido sustantivo de los textos para interpretarlos y generar construcciones discursivas ${ }^{12}$ que resulten en la categorización de las distintas fuentes de información, tales como leyes, decretos, sentencias de la corte constitucional, guías ministeriales, tesis de maestría y artículos de investigación. Más específicamente, entre los documentos relevantes estudiados se encuentran la Constitución Política de 1991, la Ley general de educación 115 de 1994, el Decreto 1860 de 1994, la jurisprudencia de la Corte Constitucional, la Ley 1620 de 2013 y su Decreto 1965, así como también la Guía ministerial 49. Las categorías encontradas y desarrolladas

8 Fierro-Evans y Carbajal-Padilla, "Convivencia escolar: una revisión del concepto".

9 Fierro-Evans y Carbajal-Padilla, "Convivencia escolar: una revisión del concepto".

${ }^{10}$ Ministerio de Educación Nacional, "Guía No. 49”.

${ }^{11}$ Ministerio de Educación Nacional, Política educativa para la formación escolar en la convivencia (Bogotá: Ministerio de Educación Nacional, 2013).

${ }^{12}$ Tania Peña Vera y Johann Pirela Morillo, "La complejidad del análisis documental”, Información, cultura y sociedad. No. 16 (2007). 
fueron las concepciones sobre la convivencia escolar que se expresan en las políticas a partir de la Constitución de 1991, así como, también, los enfoques que orientan a Colombia en el contexto de América Latina. Se encuentra que la normatividad colombiana no desarrolla una perspectiva clara respecto al papel de las familias en la convivencia escolar, a pesar de su función determinante en el aprendizaje de las competencias ciudadanas básicas. Los lineamientos se analizan en su forma de comprender el problema, los propósitos y alternativas planteadas, según los enfoques de comprensión de Morales y López. ${ }^{13}$ En seguida, se desarrolla en detalle el análisis de las categorías.

\section{Las Políticas de Convivencia Escolar desde la Constitución colombiana de 1991}

En Colombia tienen como fin asumir los principios constitucionales y objetivos misionales enfocados a la formación para el ejercicio de la paz como derecho y deber fundamental. Se parte de la Constitución de 1991 y, por su carácter, tienen que ver con la educación como derecho de la persona y servicio público a prestar de forma eficiente para todos los habitantes del territorio, ${ }^{14}$ con función social orientada a formar en el respeto a los derechos humanos, la paz y la democracia. ${ }^{15}$ Para ello, se ordena la instrucción cívica y el fomento de la participación ciudadana, ${ }^{16}$ el libre desarrollo de la personalidad ${ }^{17} \mathrm{y}$ el debido proceso, ${ }^{18}$ donde los derechos de los niños prevalecen sobre los derechos de los demás, ${ }^{19}$ principios que, además de guiar el currículo, son referentes normativos en los pactos de convivencia.

El interés del Estado en formar para el ejercicio de los derechos y la participación ciudadana se establece como uno de los fines de la educación en la Ley 115 de 1994. El Artículo $5^{\circ}$ requiere infundir el respeto a la vida,

\footnotetext{
${ }^{13}$ Macarena Morales y Verónica López, "Políticas de convivencia escolar en América Latina: cuatro perspectivas de comprensión y acción", Archivos analíticos de políticas educativas. Vol. 27. No. 5 (2019).

${ }^{14}$ Asamblea Nacional Constituyente, Constitución Política de Colombia (Bogotá: Congreso de la República, 1991), Artículo 365.

${ }^{15}$ Asamblea Nacional Constituyente, "Constitución Política de Colombia", Artículo 67.

${ }^{16}$ Asamblea Nacional Constituyente, "Constitución Política de Colombia", Artículo 47.

${ }^{17}$ Asamblea Nacional Constituyente, "Constitución Política de Colombia", Artículo 16.

${ }^{18}$ Asamblea Nacional Constituyente, "Constitución Política de Colombia", Artículo 19.

${ }^{19}$ Asamblea Nacional Constituyente, "Constitución Política de Colombia”, Artículo 44.
} 
la paz, la democracia, el pluralismo, la justicia, solidaridad, equidad, así como el ejercicio de la tolerancia y la libertad. Con esto se da a la escuela la tarea de educar para la convivencia y se plantean dos objetivos específicos: desarrollar la personalidad y la capacidad de asumir con responsabilidad y autonomía los derechos y deberes, así como, también, proporcionar una sólida formación ética, moral y la práctica de los derechos humanos. Aunque estos propósitos son misión de todo el sistema, los Artículos 20 y 21 enfatizan en la importancia de la educación básica y, más específicamente, en el ciclo de educación básica primaria, donde se pide fomentar los valores para la convivencia en una sociedad diversa.

En resumen, las políticas de convivencia le asignan a la escuela nada menos que la labor de formar la personalidad a través de la moral, la ética y los valores humanos, propósito que se encomienda de manera especial al ciclo de primaria. Veintiséis años después de formulada la meta, las instituciones educativas parecen tener problemas para cumplir con la tarea; así, por ejemplo, en un estudio desarrollado en la ciudad de Pasto, se encontró que la prevalencia de implicación en bullying fue del $41,9 \%$ y para ciberbullying del $18,7 \%^{20}$; por su parte, en una investigación desarrollada en las ciudades de Armenia y Manizales, se encontró que el consumo de drogas dentro del colegio es del $23 \%$ y $16,3 \%$, mientras que las agresiones son del $45 \%$ y $64 \%$ respectivamente; ${ }^{21}$ a su vez, en un diagnóstico desarrollado en la región Caribe, el 59,7\% de los docentes reportan que la violencia entre iguales es la forma de resolución de conflictos; más del 70\% de los profesores consideraron que se ejerce bullying hacia las personas en situación de discapacidad; el 65\% refiere que dentro del colegio se presentan problemas de microtráfico y el 55\% afirma conocer situaciones de drogodependencia en los alumnos. ${ }^{22}$

A partir de los resultados de las investigaciones, resultaría difícil pensar que las instituciones educativas desarrollan acciones equivocadas

\footnotetext{
${ }^{20}$ Mauricio Herrera López, Eva Romera y Rosario Ortega Ruiz, "Bullying y ciberbullying en Colombia; coocurrencia en adolescentes escolarizados, Revista Latinoamericana de Psicología. No. 49 (2017).

${ }^{21}$ Leonardo Alberto Vega Umbasía, Álvaro Alfonso Fernández Gallego y Néstor Raúl Giraldo Navia, "Los entornos sociales y su relación con el conflicto y la violencia escolar en las ciudades de Armenia y Manizales, Colombia", Sophia. Vol. 13. No. 1 (2017).

${ }^{22}$ Levis Yamira Cabrales Villalba et al., "Problemáticas de convivencia escolar en las instituciones educativas del caribe colombiano: análisis desde la pedagogía social para la cultura de paz". (Tesis de maestría, Universidad del Norte, 2017).
} 
o negligentes en lo que a sus propósitos misionales se refiere; más bien, se podría decir que su influencia en la estructuración de la personalidad y de sólidos principios éticos resulta ser parcial. Probablemente un actor determinante se encuentra ausente en la política: se trata de la familia que, si bien es cierto la reconoce la Ley 115 como núcleo fundamental de la sociedad y primer responsable de la educación de los hijos, de forma contradictoria se observa que la misma normativa les limita a ser colaborativas con las instituciones; de esta forma, no responden al rol que realmente tienen como agentes de formación. Ejemplo de ello es el Artículo $7^{\circ}$, donde les requiere matricular en un establecimiento, participar en las asociaciones, informarse sobre el rendimiento, contribuir solidariamente y proporcionarles un ambiente adecuado, que promueva el desarrollo integral. Vale la pena cuestionar: ¿la escuela realmente puede edificar la personalidad de los sujetos o el ambiente familiar logra ser más influyente?

Las responsabilidades de los diferentes actores de la comunidad educativa se consignan en los pactos de convivencia. La Ley 115 demanda a las instituciones educativas de un proyecto ${ }^{23}$ y un manual, en el que se definieran los derechos y obligaciones de los estudiantes,${ }^{24}$ se incluyeran las situaciones que afectan la convivencia escolar, el ejercicio de los derechos humanos, los protocolos de atención y las estrategias pedagógicas para socializar los contenidos. ${ }^{25}$ El Artículo 17 del Decreto 1860 de 1994 explicita los aspectos que debe de contemplar el reglamento: 1) reglas de higiene y salud pública; 2) criterios de respeto, valoración, compromiso frente a la utilización, conservación de bienes personales y uso colectivo; 3) pautas de comportamiento en relación con el cuidado del medio ambiente escolar; 4) normas de conducta de alumnos y profesores; 5) procedimientos para resolver con oportunidad y justicia los conflictos individuales y colectivos; 6) presentación personal, entre otros.

De igual forma, las orientaciones dadas por la Corte Constitucional son el soporte jurídico de los pactos institucionales. ${ }^{26}$ Así, por ejemplo, la sentencia T-386 de 1994 manifiesta que no es posible entrar a regular aspectos que de alguna manera afectasen los derechos constitucionales fundamentales de los

${ }^{23}$ Congreso de la República, Ley 115 de febrero 8 de 1994 (Bogotá: Congreso de la República, 1994), Artículo 73.

${ }^{24}$ Congreso de la República, "Ley 115 de febrero 8 de 1994", Artículo 87.

${ }^{25}$ Diego Cortés, “ABC de los Manuales de Convivencia”, Publicaciones semcucuta (2016).

${ }^{26}$ Cortés, "ABC de los Manuales de Convivencia". 
educandos. La SU-642 de 1998, T-688 de 2005, T-345 de 2008 y T-356 de 2013 defienden el derecho al libre desarrollo de la personalidad; T-435 de 2002, al debido proceso, que debe contemplar: 1) comunicación formal de la apertura del proceso disciplinario; 2) formulación de los cargos; 3) el traslado al imputado de todas las pruebas; 4) indicación de un término durante el cual el acusado pueda formular sus descargos; 5) el pronunciamiento definitivo de las autoridades competentes; 6) incorporación de una sanción provisional; 7) posibilidad de que el imputado pueda controvertir las decisiones de las autoridades competentes. Adicionalmente, la jurisprudencia citada sugiere tener en cuenta, para el debido proceso: 1) la edad del infractor y su grado de madurez psicológica; 2) el contexto que rodeó la falta; 3 ) las condiciones personales y familiares del alumno; 4) la existencia o no de medidas de carácter preventivo; 5) los efectos prácticos que la sanción le traerá al estudiante para su futuro educativo.

El avance de los pactos de convivencia en el ejercicio de derechos ha sido impulsado por el activismo judicial que ha tenido la Corte y no por iniciativa propia de los gobiernos ni mucho menos de las entidades educativas del sector público o privado. ${ }^{27}$ Además, se puede decir que las sentencias son los antecedentes de la Ley 1620 de 2013. Al respecto, el Artículo 21 determina que los manuales deben identificar formas alternativas para aprender del error, respetar la diversidad y dirimir los conflictos de manera pacífica. Esto significa que la política buscó la transición del reglamento como dispositivo disciplinario orientado a regular el actuar de los estudiantes, ${ }^{28}$ para convertirse en una oportunidad pedagógica de educar a los ciudadanos en los valores de la república, tales como la libertad, la democracia y justicia, donde los adultos abandonan relaciones de poder para reconocer a los menores en su dignidad.

La Ley 1620 fortalece el espíritu democrático de gestión de la convivencia a través de la conformación de los Comités nacional, municipal y escolar. Este último es de especial importancia, en tanto tiene a su cargo la ejecución operativa de la política nacional; el Artículo 13 le otorga la

${ }^{27}$ José Alejandro Ruiz Forero, "Marco legal y jurisprudencial del acoso escolar en Colombia. 10 años de jurisprudencia constitucional", Revista Temas Socio Jurídicos. Vol. 36. No. 71 (2016).

${ }^{28}$ Fernando Valencia Murcia y Viviana Mazuera, "La figura del manual de convivencia en la vida escolar. Elementos para su comprensión", Revista científica Guillermo de Ockam. Vol. 4. No. $1(20 \mid 06)$. 
función de atender conflictos por solicitud de cualquiera de los miembros de la comunidad educativa, o de oficio, cuando se estime conveniente en procura de evitar perjuicios irremediables. Según esta reglamentación, es el responsable de generar y ejecutar iniciativas a través de los proyectos pedagógicos. De igual manera, determina los componentes de la ruta, al establecer los niveles de promoción, prevención, atención y seguimiento. Cabe decir que el Comité ofrece una visión participativa de la gestión de la convivencia, no como mecanismo punitivo, sino como dirección pedagógica que promueve y potencia el pleno ejercicio del derecho a la paz; para ello, se crean los protocolos de manejo de situaciones, establecidos en el Decreto 1965, de 2013.

E1 Decreto 1965 discrimina tres tipos de situaciones y, de acuerdo con ello, establece la ruta a seguir de conformidad con los Artículos 42, 43 y 44. Las situaciones tipo I son aquellas referidas a conflictos manejados inadecuadamente y requieren: 1) reunir inmediatamente a las partes involucradas, 2) fijar la forma de solución de manera imparcial, encaminada a buscar la reparación de los daños, 3) realizar el seguimiento del caso y de los compromisos. Las situaciones tipo II tienen que ver con acoso escolar, donde se necesita: 1) garantizar la atención inmediata en salud si hay daños al cuerpo, 2) remitir a las autoridades administrativas para restablecimiento de derechos; 3) adoptar medidas de protección, 4) informar de manera inmediata a los padres o acudientes y 5) generar espacios para que las partes pudieran exponer los hechos. Por su parte, las situaciones tipo III tienen que ver con la incidencia de delitos y se debe tener en cuenta: 1) en casos de daño al cuerpo, se debe garantizar la atención inmediata en salud física y mental, 2) informar a los padres, madres o acudientes, 3) el presidente del Comité de Convivencia pondrá la situación en conocimiento de la policía nacional y 4) se establecen medidas para proteger a la víctima.

La Guía 49, del MEN, ${ }^{29}$ fundamentada en la Ley 1620 y su Decreto reglamentario, brinda una explicación detallada sobre los pasos a seguir en cada uno de los componentes de la ruta de atención integral. Como actividades de promoción, sugiere: 1) movilización de personas, formas de pensar; 2) formulación de políticas institucionales; 3) desarrollo de iniciativas y proyectos transversales. Como tareas de prevención, propone: 1) identificación de factores de riesgo y protección; 2) construcción de

\footnotetext{
${ }^{29}$ Ministerio de Educación Nacional, "Guía No. 49”.
} 
estrategias pedagógicas; 3) comunicación y manejo de información. Por su parte, la atención de situaciones se desarrolla en dos ejes: 1) el seguimiento del establecimiento educativo y 2) el trabajo intersectorial y en casa.

Seis años después de promulgada la Ley 1620, el gobierno colombiano reconoce la complejidad de problemáticas específicas que afectan la convivencia, como la relativa al consumo de sustancias psicoactivas; entonces, adopta la política integral a través de la Resolución 089, de 2019. Específicamente, en lo concerniente al campo de la educación, requiere el fortalecimiento del entorno mediante acciones como el respeto de la diversidad, las pautas de crianza, la construcción de planes o proyectos de vida y la prevención de la deserción escolar. Esto es disminuir imaginarios sociales asociados con el estigma y discriminación de los menores con trastornos mentales o problemas de consumo, a fin de evitar la desescolarización; de igual forma, desarrollar las capacidades de afrontamiento en los estudiantes para rechazar esas sustancias. Lo relevante de la normativa es el reconocimiento de la familia como actor a intervenir y, al respecto, se propone desarrollar habilidades parentales para la supervisión y el cuidado, deslegitimar el ofrecimiento de psicoactivos legales o ilegales a sus hijos en el hogar y fortalecer capacidades para la gestión de riesgos relacionados con el manejo de medicamentos. Así las cosas, en la prevención $\mathrm{y}$ atención del consumo de psicoactivos, la normativa establece deberes a la escuela y busca ofrecer derechos a la familia, pero olvida que, una vez detectado el problema en el estudiante, se requiere la aceptación de la dificultad por parte del núcleo, la necesidad de buscar ayuda y, por lo tanto, el compromiso de asegurar la asistencia del menor a los servicios de salud y/o comunitarios que diera lugar. Estos aspectos necesitan explicitarse lo suficiente en el documento, como responsabilidades de la familia.

Para la política estatal, los niveles de prevención y promoción son atribuciones de las instituciones educativas, pero, de nuevo, surge el interrogante: ¿cuál es la función de las familias en la promoción de convivencia y prevención de la violencia en la escuela? El Decreto 1286, de 2005, establece normas sobre la participación de los padres en el mejoramiento de los procesos educativos; en el Artículo $3^{\circ}$, enuncia como sus deberes: cumplir con las obligaciones contraídas en el Manual de Convivencia, contribuir a la construcción de un clima de respeto y tolerancia, así como, también, acompañar el proceso educativo. Estos deberes resultan restringidos, pues les desplazan del protagonismo que en 
realidad poseen en el desarrollo de los valores ciudadanos y les convierten en un colaborador. Entonces, se trata de una política que considera a la familia un usuario de la prestación del servicio educativo y no como el actor clave, que construye, posibilita u obstaculiza los procesos de convivencia en la escuela. Cabe cuestionar: ¿cuáles son las consecuencias si no cumplen con las obligaciones mínimas? El Decreto 1286 no especifica los modos de llevar a que se cumplieran los compromisos ni establece consecuencias; de ese modo, es un referente legal enfocado en derechos, pero no en responsabilidades. ${ }^{30}$ Es más, la Ley 1098, de 2006, Capítulo primero, Artículo 39, refuerza el papel de la familia como garante del acceso al sistema escolar y colaborador con la escuela. De esa forma, se relega al principal agente formador, transmisor de capitales sociales, culturales y simbólicos, ${ }^{31}$ ejemplo y modelo de comportamiento para los niños, donde se los introduce en el mundo de las personas y las relaciones, ${ }^{32}$ primer espacio de socialización creador de las concepciones y explicaciones acerca de la convivencia y el ejercicio de los derechos. ${ }^{33}$

En un intento por reconocer la importancia de la educación familiar, la Ley 1404, de 2010, crea la Escuela de padres y madres; implantar estos proyectos es otro deber de los establecimientos, lo que operativamente está a cargo de los docentes orientadores, pero sin asignar ningún tipo de responsabilidad a las familias, ni siquiera la obligatoriedad de asistir a los encuentros. Este hecho condujo a la realización y cumplimiento de eventos por parte de los establecimientos con ausencia de los acudientes. De alguna forma, se buscó reparar el vacío en los deberes con la Ley 2025, de 2020, que faculta a los planteles para la imposición de sanciones si no se acude a las citaciones, más resulta un desafío idear consecuencias que no entrasen en conflicto con los derechos de los menores ni tampoco implicaran costos de tipo pecuniario.

${ }^{30}$ Luz Stella Fuentes y Libia María Pérez Castro, "Convivencia escolar: una mirada desde las familias", Revista de Estudios Interdisciplinarios en Ciencias Sociales. Vol. 21. No. 1 (2019).

${ }^{31}$ Milcíades Vizcaíno Gutiérrez, "La formación para la convivencia: un reto para la gestión escolar", Cuaderno venezolano de sociología. Vol. 24. No. 13 (2015).

${ }^{32}$ Claudia Patricia Cuero et al. "Propuesta de intervención psicopedagógica para el mejoramiento de la convivencia escolar en estudiantes de grado séptimo de cuatro instituciones públicas del oriente antioqueño" (Tesis de maestría, Universidad Pontificia Bolivariana, 2018).

${ }^{33}$ Fuentes y Pérez Castro, "Convivencia escolar: una mirada desde las familias". 
Probablemente el enfoque desarrollado por la legislación colombiana en lo concerniente a la enseñanza para la promoción de la convivencia proviene de creer que la institución educativa es más competente e idónea para transmitir los valores de la coexistencia que la familia. Esto se aleja de la realidad y deja ver el desconocimiento de la ciencia psicológica, así como la poca experiencia de los autores de las políticas públicas en el ejercicio docente, en tanto las investigaciones de García, ${ }^{34}$ Quiñones y Valencia, ${ }^{35}$ Salas y Cómbita, ${ }^{36}$ muestran que las condiciones específicas del ambiente familiar predisponen al individuo para la sana convivencia, en contextos como: estilos de crianza adecuados, el ejercicio de la autoridad, los límites, la libertad, la comprensión de los cambios que sufren los hijos, el desarrollo de recursos de apoyo, el aprendizaje de valores, habilidades de resolución de conflictos, la aceptación de sí mismo y de los demás, la promoción de una representación de la violencia que ayude a prevenirla.

De otro lado, en lo referente a la atención para la convivencia, la Guía ministerial $49^{37}$ ofrece una visión del conflicto renovada, no desde la perspectiva sancionatoria, sino como oportunidad para que todas las personas que conforman la comunidad educativa utilizaran el diálogo para transformar las relaciones, el pensamiento crítico como mecanismo para entender lo que ocurre, la capacidad para ponerse en los zapatos de la otra persona como forma de reconocerse y la concertación para salvar las diferencias.

Así las cosas, según el gobierno, la aparición del conflicto es una situación que se caracteriza por la incompatibilidad real o percibida entre los intereses de una o varias personas, ${ }^{38}$ que puede manifestarse mediante conversaciones, diálogos, discusiones, altercados, enfrentamientos o riñas. La Guía 49 refiere que pueden manejarse constructivamente por medio del diálogo, la negociación o la mediación. A esta última se la entiende como el proceso de resolución de conflictos en el que las partes enfrentadas acuden

${ }^{34}$ Carmen María García Escamilla. "Familia y convivencia escolar" (Tesis de maestría, Universidad de Almería, 2011).

${ }^{35}$ Edwin Davian Quiñones Rivero y Jaqueline Valencia Quintero. "Construcción de valores en la familia y convivencia escolar" (Tesis de maestría, Universidad de la Sabana, 2016).

${ }^{36}$ Karen Elena Salas Viloria y Harold Arturo Cómbita Niño, "Análisis de la convivencia escolar desde la perspectiva psicológica, legal y pedagógica en Colombia", Cultura, Educación y Sociedad. Vol. 8. No. 2 (2017).

${ }^{37}$ Ministerio de Educación, "Guía No. 49".

${ }^{38}$ Ministerio de Educación, "Guía No. 49". 
voluntariamente a una tercera persona, llamada mediador o mediadora, con el fin de llegar a un acuerdo que sea satisfactorio. ${ }^{39}$

Si bien, la Guía 49 sugiere el empleo de la mediación en las situaciones tipo I, no es lo suficientemente aprovechada en el sistema de educación colombiano de básica y media, puesto que el país no cuenta con una ley reglamentaria ni existen políticas claras para implementar un programa nacional, como ocurre en otros países de Latinoamérica, tal como el caso de Argentina, con la Ley 3055, de 2009. Pese a que este es un punto para mejorar en la política educativa colombiana, es claro que los documentos oficiales a partir del año 2013 sugieren un cambio de paradigma en los reglamentos de las instituciones educativas, que evidencia la resignificación del conflicto como oportunidad de aprendizaje, lo cual es coherente con la investigación psicológica sustentada en autores como Torrego, ${ }^{40} \mathrm{Jares},{ }^{41}$ Zambrano ${ }^{42}$ y Ortega et al. ${ }^{43}$

Por otra parte, las políticas públicas de convivencia escolar en Colombia no se limitan a la estructuración de reglamentos o la definición de protocolos. Es importante el recurso humano encargado de la gestión de la convivencia. Desde 1994, el Decreto 1860, Artículo 40, reglamenta

${ }^{39}$ Definición soportada en distintos autores: Silvia Lungman, La mediación escolar (Buenos Aires: Lugar Editorial, 1996); Rosario Ortega Ruiz y Rosario del Rey, "La mediación escolar en el marco de la construcción de la convivencia y la prevención de la violencia”, Avances en supervisión educativa: Revista de la Asociación de Inspectores de Educación de España. No. 2 (2006); María Jesús Cava Caballero, "La utilidad de la mediación como estrategia de resolución y prevención de conflictos en el ámbito escolar", Información Psicológica. No. 95 (2009); Rosa Pulido Valero et al., "La mediación escolar en la comunidad de Madrid: Análisis del impacto de la formación en el profesorado y alumnado en el IES Las Américas de Parla", Revista de Mediación. Año 3. No. 6 (2010); Laura Hermosilla Benítez, "Programa de mediación escolar y formación de alumnos" (Trabajo de master, Universidad de Valladolid, 2015); Katia Rolán González et al., "Acoso escolar en primaria tras la formación en técnicas de mediación", Revista de Estudios e Investigación en Psicología y Educación. No 2 (2017); Martha Hilda González Calderón, "Educar para la paz, una tarea de todos. Mediación escolar", Cotidiano. Vol. 33. No. 208 (2018).

${ }^{40}$ Juan Carlos Torrego, Mediación de conflictos en instituciones educativas. Manual para la formación de formadores. (Madrid: Narcea, 2000).

${ }^{41}$ Xesús R. Jares, "El lugar del conflicto en la organización escolar", Revista Iberoamericana de Educación. No. 302 (1997).

${ }^{42}$ Raquel Zambrano Ramírez, "Implantación de un programa de mediación escolar", Vía Docente. No. 1 (2015).

${ }^{43}$ Raúl Ortega Mondaca et al. Orientaciones estratégicas para la formación de monitores en mediación de conflictos escolares (Santiago de Chile: Universidad de Chile, 2007). 
los objetivos del servicio de orientación, que trabaja en pro del pleno desarrollo de la personalidad de los educandos en cuanto a la toma de decisiones personales, identificación de aptitudes e intereses, solución de conflictos y problemas individuales, familiares y grupales, participación en la vida académica, social y comunitaria, y desarrollo de valores relativos a la formación personal, que trata el Artículo 92 de la Ley 115.

El Artículo 32, de la Ley 1620, adiciona funciones a la orientación escolar respecto a la convivencia, que consisten en identificar factores de riesgo, registrar en el sistema los casos de acoso o vulneración de derechos, participar en el proceso de acompañamiento, así como de la evaluación de los resultados, actuar como agentes de enlace a las entidades según la ruta de atención integral, participar en los planes individuales y grupales de intervención, seguir los protocolos establecidos, apoyar al Comité escolar y documentar los casos, participar en la construcción, redacción, socialización e implementación del Manual de Convivencia y de los proyectos pedagógicos, involucrar a las familias a través de la Escuela de padres.

A modo de resumen y a fin de presentar con claridad la evolución de las políticas de convivencia escolar desde la Constitución de 1991, se realiza el cuadro tipológico, que incluye las principales normas, jurisprudencia y lineamientos ministeriales, así como, también, la síntesis de los avances y consideraciones.

\begin{tabular}{|l|l|l|}
\hline \multicolumn{1}{|c|}{ Documento } & \multicolumn{1}{|c|}{ Principales avances } & \multicolumn{1}{c|}{ Consideraciones } \\
\hline $\begin{array}{l}\text { Constitución } \\
\text { Política, de de } \\
1991\end{array}$ & $\begin{array}{l}\text { Establece que la educación es un } \\
\text { derecho y un servicio público, } \\
\text { ordena la instrucción cívica y el } \\
\text { fomento de los principios de la } \\
\text { democracia, tales como el libre } \\
\text { desarrollo de la personalidad y el } \\
\text { debido proceso. }\end{array}$ & $\begin{array}{l}\text { Representa un paradigma que } \\
\text { orienta la política de educación } \\
\text { el Estado de derecho. Entre otras } \\
\text { dimensiones de la vida escolar, } \\
\text { se expresa en el currículo y los } \\
\text { manuales. }\end{array}$ \\
\hline $\begin{array}{l}\text { Ley 115, de } \\
1994\end{array}$ & $\begin{array}{l}\text { Propone como fines el desarrollo } \\
\text { de valores asociados con la justicia, } \\
\text { solidaridad, equidad, tolerancia y } \\
\text { libertad. Le otorga a la escuela el } \\
\text { objetivo de formar la personalidad } \\
\text { desde un sólido criterio ético, mo- } \\
\text { ral y de convivencia. }\end{array}$ & $\begin{array}{l}\text { La Ley general de educación le } \\
\text { encarga a la escuela nada menos } \\
\text { que la tarea de formar la persona- } \\
\text { lidad de los educandos. De ahí la } \\
\text { pregunta: ila escuela realmente } \\
\text { puede edificar la personalidad de } \\
\text { los sujetos o el ambiente familiar } \\
\text { logra ser más influyente? }\end{array}$ \\
\hline
\end{tabular}




\begin{tabular}{|c|c|c|}
\hline $\begin{array}{l}\text { Decreto } 1860, \\
\text { de } 1994 .\end{array}$ & $\begin{array}{l}\text { Establece los aspectos que debe } \\
\text { contener el Manual de Convivencia. }\end{array}$ & $\begin{array}{l}\text { Propugna por la construcción } \\
\text { democrática de los reglamentos } \\
\text { escolares. }\end{array}$ \\
\hline $\begin{array}{l}\text { Decreto } 1286, \\
\text { de } 2005\end{array}$ & $\begin{array}{l}\text { Determina normas sobre la } \\
\text { participación de los padres de } \\
\text { familia en el mejoramiento de los } \\
\text { procesos educativos. }\end{array}$ & $\begin{array}{l}\text { Los deberes resultan restringidos, } \\
\text { al desconocer el importante rol que } \\
\text { posee la familia en el desarrollo } \\
\text { de actitudes prosociales, así como, } \\
\text { también, lo reducen a ser un } \\
\text { colaborador de las instituciones } \\
\text { educativas. }\end{array}$ \\
\hline $\begin{array}{l}\text { Ley } 1404 \text {, de } \\
2010\end{array}$ & $\begin{array}{l}\text { Crea el programa Escuela de pa- } \\
\text { dres y madres en las instituciones } \\
\text { de educación preescolar, básica y } \\
\text { media del país. }\end{array}$ & $\begin{array}{l}\text { Llevar a cabo este tipo de inicia- } \\
\text { tivas es una responsabilidad de } \\
\text { las instituciones educativas, cuya } \\
\text { gestión operativa está a cargo de } \\
\text { orientación escolar. Sin embargo, } \\
\text { no asigna ningún tipo de deber a } \\
\text { las familias. }\end{array}$ \\
\hline $\begin{array}{l}\text { Sentencias de } \\
\text { la Corte cons- } \\
\text { titucional }\end{array}$ & $\begin{array}{l}\text { Son referentes en la construcción } \\
\text { de los Manuales de Convivencia, } \\
\text { basados en los derechos constitu- } \\
\text { cionales fundamentales de los edu- } \\
\text { candos. }\end{array}$ & $\begin{array}{l}\text { Las sentencias de la Corte requie- } \\
\text { ren considerar, además de la falta } \\
\text { en sí, las circunstancias familiares, } \\
\text { personales y sociales que rodean al } \\
\text { estudiante en la búsqueda del ejer- } \\
\text { cicio de sus derechos. Son los ante- } \\
\text { cedentes de la Ley } 1620 \text {, de } 2013 \text {. }\end{array}$ \\
\hline $\begin{array}{l}\text { Ley } 1620 \text {, de } \\
2013\end{array}$ & $\begin{array}{l}\text { Implica la transición del reglamen- } \\
\text { to como dispositivo disciplinario } \\
\text { para constituirse en un método pe- } \\
\text { dagógico que facilita la educación } \\
\text { en la democracia. También, esta- } \\
\text { blece el Comité escolar de Convi- } \\
\text { vencia, conformado por el perso- } \\
\text { nero, representante de padres de } \\
\text { familia, de profesores, orientación } \\
\text { escolar, rectoría, entre otros esta- } \\
\text { mentos. }\end{array}$ & $\begin{array}{l}\text { No se observan lineamientos que } \\
\text { preparen al personero estudiantil } \\
\text { para la participación real en el Co- } \\
\text { mité de Convivencia. De otro lado, } \\
\text { los orientadores escolares son res- } \\
\text { ponsables de la ejecución de la ruta } \\
\text { de atención. A pesar de ello, no se } \\
\text { promueve ningún incremento de } \\
\text { los pedagogos mencionados. }{ }^{44} \text { Por } \\
\text { lo tanto, no se cuenta con los recur- } \\
\text { sos de talento humano suficientes } \\
\text { para dar cumplimiento a la norma. }\end{array}$ \\
\hline
\end{tabular}

${ }^{44}$ Leonel Martínez, "Análisis del sistema nacional de convivencia escolar y formación para el ejercicio de los derechos humanos, sexuales y reproductivos desde la perspectiva de la eficacia simbólica”, Pensamiento Jurídico. No. 49 (2018). 


\begin{tabular}{|l|l|l|}
\hline $\begin{array}{l}\text { Decreto 1965, } \\
\text { de } 2013\end{array}$ & $\begin{array}{l}\text { Especifica las acciones a seguir } \\
\text { para la atención de situaciones, } \\
\text { según su tipología: conflictos ma- } \\
\text { nejados inadecuadamente (Tipo I), } \\
\text { acoso escolar (Tipo II), presuntos } \\
\text { delitos (Tipo III). }\end{array}$ & $\begin{array}{l}\text { El Ministerio de Educación no } \\
\text { asume responsabilidades en la eje- } \\
\text { cución de la ruta de atención, toda } \\
\text { vez que no asigna un presupuesto } \\
\text { específico para cumplir con las fi- } \\
\text { nalidades de la ley. }\end{array}$ \\
\hline $\begin{array}{l}\text { Guía } 49, \\
\text { MEN 2014 }\end{array}$ & $\begin{array}{l}\text { La guía sugiere el empleo de la me- } \\
\text { diación en los conflictos manejados } \\
\text { inadecuadamente (situaciones tipo } \\
\text { I). }\end{array}$ & $\begin{array}{l}\text { La mediación no es lo suficiente- } \\
\text { mente aprovechada en el sistema } \\
\text { de educación colombiano de bá- } \\
\text { sica y media, ya que el país no } \\
\text { cuenta con una ley reglamentaria } \\
\text { ni existen políticas claras para im- } \\
\text { plementar un programa nacional } \\
\text { de mediación. }\end{array}$ \\
\hline $\begin{array}{l}\text { Ley 2025, de } \\
2020\end{array}$ & $\begin{array}{l}\text { Establece lineamientos para la im- } \\
\text { plementación de las escuelas de fa- } \\
\text { milia en términos de frecuencia y } \\
\text { contenidos. }\end{array}$ & $\begin{array}{l}\text { A pesar de determinar obligato- } \\
\text { riedad, no ofrecen garantías a las } \\
\text { familias para asistir a los encuen- } \\
\text { tros, toda vez que hay ausencia de } \\
\text { alternativas para obtener permiso } \\
\text { laboral. }\end{array}$ \\
\hline
\end{tabular}

Tabla 1. Evolución de las Políticas de Convivencia Escolar, desde la Constitución de 1991

Fuente: elaboración de esta investigación

\section{Enfoques de las Políticas de Convivencia Escolar colombianas en el contexto de América Latina}

Si bien definir la convivencia escolar representa una tarea de alta complejidad, por cuanto aún es objeto de construcción, ${ }^{46}$ en los Estados de América Latina parecen existir consensos en torno a su acepción.

${ }^{45}$ Martínez, "Análisis del sistema nacional de convivencia escolar y formación para el ejercicio de los derechos humanos, sexuales y reproductivos desde la perspectiva de la eficacia simbólica".

${ }^{46}$ Antonieta Aguilera García, "Desafíos conceptuales y metodológicos para evaluar la convivencia escolar", en Reflexiones sobre convivencia escolar. De las políticas públicas al salón de clases, coords. Azucena Ochoa Cervantes y José Juan Salinas de la Vega (Querétaro: Universidad Autónoma de Querétaro, 2018). 
Por ejemplo, los Ministerios de Educación de Colombia ${ }^{47}$, Chile ${ }^{48}$ y Perú $^{49}$ la definen como el conjunto de relaciones interpersonales que dan forma a una comunidad educativa y se construyen de modo colectivo y cotidiano en corresponsabilidad. En especial, Colombia y Perú equiparan la convivencia al ejercicio de los derechos humanos, la armonía y el desarrollo integral de los estudiantes. Por su parte, el Ministerio de Educación de Chile $^{50}$ amplía el espectro, al dejar en claro que este concepto no se reduce a los elementos prosociales, pero tampoco a la violencia o a las agresiones. Por lo tanto, incluye tanto a las dimensiones saludables como a las no saludables producidas en el lazo social.

Las acepciones de la convivencia propuestas por los gobiernos son coherentes con los avances teóricos de prestigiosos investigadores latinoamericanos; incluso se puede decir que se nutren de los aportes realizados por la academia. Por ejemplo, Carbajal y Fierro ${ }^{51}$ argumentan que este concepto tiene un sentido amplio, cuya naturaleza se enfoca a construir paz a partir de prácticas pedagógicas, de gestión inclusiva, equitativa y participativa, que permitieran abordar de forma constructiva los conflictos en los ámbitos de la vida escolar, pedagógico, curricular, organizativo administrativo y socio-comunitario.

La forma como se entiende el objeto orienta las intervenciones de la política pública; esto queda explícito en la legislación de cada país y, a su vez, trae un enfoque de abordaje. En Latinoamérica, se establecen leyes de violencia y convivencia escolar que obedecen a los lineamientos emanados de los organismos internacionales, como el Banco Mundial y la Organización para la Cooperación y el Desarrollo Económico (OCDE), que presionan por resultados medibles. ${ }^{52} \mathrm{Al}$ tomar en cuenta el contexto subyacente, cuatro perspectivas orientan las políticas de convivencia en

${ }^{47}$ Ministerio de Educación Nacional, "Guía No. 49".

${ }^{48}$ Ministerio de Educación, Chile, Política Nacional de Convivencia Escolar (Santiago de Chile: Ministerio de Educación, 2019).

${ }^{49}$ Presidencia de la República, Perú. Lineamientos para la Gestión de la Convivencia Escolar, la Prevención y la Atención de la Violencia contra Niñas, Niños y Adolescentes (Lima: Diario Oficial El Peruano, 2018).

${ }^{50}$ Ministerio de Educación, Chile, "Política Nacional de Convivencia Escolar".

${ }^{51}$ Fierro-Evans y Carbajal-Padilla, "Convivencia escolar: una revisión del concepto".

${ }^{52}$ Verónica López et al., "Editorial Sección Temática Violencia y Convivencia en América Latina: Políticas, prácticas, marcos de comprensión y acción”, Psicoperspectivas. Vol. 18. No. 1 (2019). 
Latinoamérica: democrática, de seguridad ciudadana, de salud mental infanto-juvenil y managerialista. ${ }^{53}$

La perspectiva democrática concibe a la convivencia en la escuela como el cultivo de la vida colectiva, caracterizada por un currículo con énfasis en la paz, la ciudadanía, los derechos humanos, la creación de estructuras de participación y el reconocimiento de la diversidad; ejemplo de esta perspectiva son los lineamientos de Costa Rica, Venezuela y Uruguay. Las estrategias de seguridad ciudadana corresponden a mecanismos de control normativo y punitivo de la conducta de los estudiantes que generan parámetros de disciplina escolar, tal como es el caso de Cuba, República Dominicana, Guatemala y Paraguay. El enfoque de salud mental busca garantizar la implementación de la Convención de los Derechos del Niño respecto a proveer de condiciones para el desarrollo saludable, libres de violencia y discriminación, como ocurre en Honduras, Ecuador y Argentina. Para la concepción managerialista, la convivencia es un estado de disciplina y armonía en la escuela, basado en la gestión institucional racional y estratégica, como es el caso de Perú. ${ }^{54}$

Se puede decir que dos de las perspectivas descritas en Ascorra et al. ${ }^{55}$ así como, también, en Morales y López, ${ }^{56}$ son análogas a los enfoques encontrados por Carbajal y Fierro, ${ }^{57}$ de modo tal que la concepción democrática es equivalente a la educación para la ciudadanía y para los derechos humanos; la de salud mental infanto-juvenil a la educación para la paz, el desarrollo moral y la formación en valores. La perspectiva de seguridad ciudadana y managerialista son enfoques no incluidos en Carbajal y Fierro. En seguida, se presentan las políticas de convivencia escolar colombianas, en el contexto de los enfoques agenciados en América Latina.

\footnotetext{
${ }^{53}$ Enfoques propuestos por los autores: Paula Ascorra Costa, Verónica López Leiva y Macarena Morales, "Políticas públicas en convivencia escolar. Una visión comparada en América Latina: El caso de Chile", en Reflexiones sobre convivencia escolar. De las politicas públicas al salón de clases, eds. Azucena Ochoa Cervantes y Juan José Salinas de la Vega (México: Universidad Autónoma de Queretano, 2018); Macarena Morales y Verónica López, "Políticas de convivencia escolar en América Latina: cuatro perspectivas de comprensión y acción", Archivos analíticos de políticas educativas. Vol. 27. No. 5 (2019).

${ }^{54}$ Presidencia de la República, Perú. "Lineamientos para la Gestión de la Convivencia Escolar, la Prevención y la Atención de la Violencia contra Niñas, Niños y Adolescentes".

${ }^{55}$ Ascorra Costa, López Leiva y Morales, "Políticas públicas en convivencia escolar".

${ }^{56}$ Morales y López, "Políticas de convivencia escolar en América Latina".

${ }^{57}$ Fierro-Evans y Carbajal-Padilla, "Convivencia escolar: una revisión del concepto".
} 
Colombia muestra una tendencia a la perspectiva democrática, al buscar potenciar las capacidades de los estudiantes para la participación; ya desde 2004 el Ministerio de Educación implementa en el currículo las competencias ciudadanas explicitadas en la Guía ministerial seis; ${ }^{58}$ en 2008, promulga la Guía educación para la sexualidad, ${ }^{59}$ inspirada en la igualdad de género, así como, también, en la vivencia de los derechos sexuales y reproductivos; en 2013, la Ley 1620 crea el Comité de Convivencia escolar para generar representación de todos los actores educativos, de ahí que los Manuales de Convivencia usualmente reglamentan prácticas concertadas para la aplicación de estímulos, como, también, de medidas correctivas. Entonces, Colombia parte de un enfoque de derechos, pero también asume elementos propios de seguridad ciudadana, en tanto la autonomía institucional dada por la Ley 115 otorga la facultad para establecer pactos, que incluyen los deberes y las sanciones por no cumplimiento, siempre que se tengan en cuenta los principios constitucionales del debido proceso, defensa y educación. ${ }^{60}$

Ahora bien, dentro del modelo que orienta la política de convivencia escolar colombiana, se inserta la influencia del enfoque de salud mental. Así, por ejemplo, la Ley de infancia y adolescencia traza un marco de referencia esencialmente pedagógico, constructivo y no sancionatorio, de tal manera que las situaciones tipo II y III debe atenderlas el sistema salud y es responsabilidad de los establecimientos realizar el respectivo reporte, tal como lo establece el Decreto 1965, de 2013. De esa forma, se teje la articulación interinstitucional, donde los niveles de promoción y prevención los desarrollan las instituciones educativas a través de iniciativas enmarcadas en los proyectos transversales; por su parte, los niveles de atención y seguimiento demandan al sector salud la intervención del caso. Si bien el protocolo requiere convocar a Comité en las situaciones tipo II y III, las disposiciones legales no son lo suficientemente claras sobre las acciones a tomar para la gestión de la seguridad institucional, ${ }^{61}$ más aún cuando la escuela debe darse su propia organización e involucrar la

${ }^{58}$ Enrique Chaux, coord., Estándares Básicos de Competencias Ciudadanas. Formar para la ciudadanía ... isí es posible! (Bogotá: Ministerio de Educación Nacional, 2004).

${ }^{59}$ Ministerio de Educación Nacional. Guía No. 1. Educación para la sexualidad y construcción de ciudadanía (Bogotá: Ministerio de Educación, 2008).

${ }^{60}$ Ministerio de Educación Nacional. Concepto ER-027640. Asunto: Sanciones del Manual de Convivencia. (Bogotá: Ministerio de Educación Nacional, 2016).

${ }^{61}$ María Cecilia Fierro-Evans, "Convivencia inclusiva y democrática. Una perspectiva para gestionar la seguridad escolar”, Sinéctica, revista electrónica de educación. No. 40 (2013). 
participación de los distintos estamentos, como son los alumnos, docentes, directivos y padres y madres de familia.

Por último, según Morales y López, ${ }^{62}$ la concepción managerialista es la más ecléctica de las cuatro y propone como ejemplo a Colombia, que implementó la certificación de calidad de los establecimientos con base en la evaluación de ítems de convivencia escolar. Es probable que este sistema fuese parte de la política, pero no es suficiente para definirlo dentro de dicha corriente; una lectura más detallada devela que desde la Constitución de 1991 se visualiza la función de la educación para la vida en sociedad desde los principios de la república, como participación, libertad, justicia y dignidad. Por lo tanto, el enfoque democrático inspira la política de convivencia escolar colombiana.

\section{Conclusiones}

Así, las políticas públicas de convivencia en Colombia parten de la educación como derecho fundamental de los niños, ${ }^{63}$ con el objeto de formar la personalidad a través de una sólida enseñanza ética y moral que se imparte en las instituciones educativas y con especial énfasis en el ciclo de educación básica primaria. ${ }^{64}$ Para ello, se requiere de un proyecto, como, también, de un manual que, debido al avance de la jurisprudencia, el actuar de la Corte Constitucional y mediante la Ley 1620 y su Decreto reglamentario incorpora la ruta de atención como parte fundamental. Los propósitos y modos de atender la problemática parten de la concepción democrática, derechos humanos y educación para la paz, donde se atienden situaciones específicas desde el sistema de salud como parte del disfrute de derechos; los lineamientos ofrecen, también, la posibilidad de adoptar medidas correspondientes a la visión de seguridad ciudadana (a través de las sanciones) y managerialista en el sistema de acreditación de calidad.

Al respecto, Bocanegra y Herrera $^{65}$ aseguran que la política de convivencia, en el plano teórico, responde al requerimiento del

\footnotetext{
${ }^{62}$ Morales y López, "Políticas de convivencia escolar en América Latina: cuatro perspectivas de comprensión y acción".

${ }^{63}$ Asamblea Nacional Constituyente, Constitución política, Artículo 44.

${ }^{64}$ Congreso de la República, Ley 115, Artículo 20.

${ }^{65}$ Henry Bocanegra Acosta y Carlos Herrera Castillo, "La ley 1620 de 2013 y la política pública educativa de convivencia escolar en Colombia: entre la formalidad jurídica y la realidad social", Revista Republicana. No. 23 (2017).
} 
sector educativo para atender el creciente fenómeno de violencia al interior de los establecimientos $\mathrm{y}$, en algunos casos, los resultados son los procesos de ajuste en los manuales e incluso el impacto sobre el currículo y el proyecto educativo; sin embargo, "en la mayoría de las instituciones no se ha pasado de un ejercicio formal que no ha tenido ningún impacto". ${ }^{66}$ Con todo, la Ley 1620 representa un referente para los programas de mejoramiento, bien por iniciativa de cada establecimiento, bien por desarrollo teórico de los académicos. Ejemplo de ello son las investigaciones de Guerrero y Cepeda ${ }^{67}$ quienes parten de la fundamentación jurídica para proponer estrategias que facilitasen la reinserción de estudiantes desplazados por la violencia al sistema educativo urbano. También, el texto de Castellanos y Pérez, ${ }^{68}$ quienes, motivados en el espíritu pedagógico de la norma, reafirman la importancia de implementar estrategias de gestión pacífica de conflictos en las aulas a través de la mediación.

Como producto de mi experiencia laboral en instituciones educativas, se observa que, en la práctica, los docentes orientadores y coordinadores se enfocan más en las actividades de atención y seguimiento que en la promoción y prevención. Estas actividades usualmente se intencionan desde el método sanción y mecanismo del debido proceso posterior a todo un proceso de diálogo y firmas de compromisos. ${ }^{69}$ Así las cosas, el abordaje de la convivencia realizado por las instituciones educativas se mantiene intacto pese a la evolución de las distintas reformas, que exigen el desarrollo de proyectos, programas y estrategias sin que el Ministerio

${ }^{66}$ Bocanegra Acosta y Herrera Castillo, "La ley 1620 de 2013 y la política pública educativa de convivencia escolar en Colombia", p. 209.

${ }^{67}$ Hilda Rosa Guerrero Cuentas y María Luz Cepeda, "Uso de estrategias pedagógicas para el fortalecimiento de la convivencia escolar de jóvenes vulnerables", Revista de pedagogía. Vol. 37. No. 101 (2016).

${ }^{68}$ Flor Liliana Castellanos Bothia y Karen Estefanni Pérez Álvarez, "La convivencia escolar: una arista de paz en medio del posconflicto", Temas socio-jurídicos. Vol. 38. No. 76 (2019).

${ }^{69}$ Hallazgos en las investigaciones de: Ivette Andrea Castro Jiménez, "Propuesta para la implementación de la mediación escolar como método alternativo de resolución de conflictos para la construcción de una convivencia pacifica en las instituciones educativas públicas de la ciudad de Pasto" (Trabajo final de especialización, Universidad de Nariño, 2013); Claudia Patricia Cuero, Sandy Leandra Garcés, Andrés Mauricio Iral y Adriana Patricia Suárez, "Propuesta de intervención psicopedagógica para el mejoramiento de la convivencia escolar en estudiantes de grado séptimo de cuatro instituciones públicas del oriente antioqueño" (Tesis de maestría, Universidad Pontificia Bolivariana, 2018). 
de Educación garantizara los recursos financieros y de talento humano para este propósito. ${ }^{70}$

La tendencia a la aplicación del método sanción y, en menor medida, al despliegue de proyectos de prevención y promoción (hechos que caracterizan un régimen autoritario escolar), probablemente se relaciona con las condiciones laborales que las autoridades públicas establecen, en especial las alusivas al Decreto 1850 de 2002 y a las relaciones técnicas docente, orientador y directivo por número de estudiantes, circunstancias que dificultan las labores grupales, focalizadas o personalizadas, requeridas en el fortalecimiento de la convivencia. También, a causa del número de situaciones que diariamente se presentan, que no se desligan de las condiciones sociales que lo determinan, en ese sentido los desarrollos jurídicos son insuficientes si no se atiende la problemática socioeconómica $\mathrm{y}$ de seguridad que afrontan los estudiantes y sus familias, ${ }^{71}$ así como, también, si las leyes no se soportan en recursos financieros que den lugar a su cumplimiento.

Aún falta claridad en la norma y en los lineamientos ministeriales respecto a las estrategias para prevenir, atender la violencia en las escuelas y promover la convivencia desde los ejes de gestión institucional que proponen Fierro ${ }^{72}$ y Chaparro y Mora ${ }^{73}$. Entonces, las instituciones deben ir más allá de la Guía ministerial $34^{74}$ para realizar su autoevaluación y cuestionar la forma como se reconocen y atienden las necesidades de las otras personas, se valora la diversidad, se genera participación, se promueve el diálogo, se desarrolla un trato respetuoso y considerado. ${ }^{75}$

Más específicamente, supone focalizar esfuerzos de los establecimientos en el desarrollo de programas, no como emprendimientos exclusivos de

${ }^{70}$ Leonel Martínez, “Análisis del sistema nacional de convivencia escolar y formación para el ejercicio de los derechos humanos, sexuales y reproductivos desde la perspectiva de la eficacia simbólica", Pensamiento Jurídico. No. 49 (2018).

${ }^{71}$ Bocanegra Acosta y Herrera Castillo, "La ley 1620 de 2013 y la política pública educativa de convivencia escolar en Colombia".

${ }^{72}$ María Cecilia Fierro-Evans, "Convivencia inclusiva y democrática".

${ }^{73}$ Alicia Aleli Chaparro Caso y Noé Mora Osuna, "Desafíos de la evaluación de la convivencia escolar", en Reflexiones sobre convivencia escolar. De las políticas públicas al salón de clases.

${ }^{74}$ James Valderrama Rengifo. Guía No. 34. Guía para el Mejoramiento Institucional. De la Autoevaluación al Plan de Mejoramiento (Bogotá: Ministerio de Educación Nacional, 2008).

${ }^{75}$ Fierro-Evans, "Convivencia inclusiva y democrática". 
los orientadores o coordinadores, sino como políticas institucionales que abordasen la complejidad del fenómeno como sistema. Dicho en otras palabras, se pretende generar un verdadero trabajo en equipo y por proyectos, pero, primero, se necesita deconstruir las dinámicas organizacionales que tradicionalmente generan división y sectores, que se manifiestan en docentes exclusivos del área académica, aquellos dedicados a la formación para el trabajo, el rector entregado por completo a la dimensión administrativofinanciera y el orientador como único responsable de ejecutar la ruta de atención. El resultado del mecanicismo con el que funcionan las instituciones educativas poco aporta a la disminución de la incidencia de situaciones que por protocolo deben remitirse a entidades de salud o a Bienestar Familiar (ICBF), según fuese el caso. Tal como lo exponen Bocanegra y Herrera, ${ }^{76} \mathrm{el}$ problema consiste en que se genera sobresaturación y, con ello, dificultades para la prestación de servicios en salud, demoras en la programación de citas por falta de agenda, igual ocurre en ICBF, donde el número de casos desborda las capacidades y se dificulta la oportuna intervención y apoyo para los estudiantes.

De otro lado, resulta preocupante cómo las políticas de convivencia escolar en Colombia descuidan el papel de las familias en el conflicto y se relega su rol al de acompañantes de las instituciones. Si bien se reconoce como núcleo fundamental de la sociedad, de forma contradictoria no se dejan explícitos los medios para llevarla a que cumpliera con su deber como principal agente de formación educativa. ${ }^{77}$ Más aún si se tiene en cuenta que moldea el desarrollo del niño; es el lugar donde se aprenden los valores, cuyas relaciones influirán en los modos de solucionar los conflictos. ${ }^{78}$ Por lo tanto, es conveniente revisar los mensajes que envían los padres a sus hijos en lo concerniente a los métodos para responder a la afrenta. Si los padres son referentes en el desarrollo de los menores, es menester de la legislación y las políticas educativas abordar el rol de la familia en la promoción de la convivencia escolar.

Las reflexiones consignadas en este artículo conducen a proponer futuras líneas de investigación asociadas con: 1) diagnóstico de la

\footnotetext{
${ }^{76}$ Bocanegra Acosta y Herrera Castillo, "La ley 1620 de 2013 y la política pública educativa de convivencia escolar en Colombia".

${ }^{77}$ Salas Viloria y Cómbita Niño, "Análisis de la convivencia escolar desde la perspectiva psicológica, legal y pedagógica en Colombia".

${ }^{78}$ Fanny Esperanza Ramírez Munar, Fanny Esperanza y Guiovanna Ramírez Ospina. "Propuesta pedagógica con enfoque relacional para fortalecer los vínculos familiares y mejorar la convivencia" (Tesis de Maestría, Universidad de la Sabana, 2016).
} 
convivencia escolar resultado de las diferentes reformas educativas; 2) estudio de la aplicación concreta de las políticas de convivencia en los planteles educativos para analizar las carencias de la normativa y del propio centro en la implementación; 3) análisis de la política para identificar si se diseña y construye colectivamente, así como, también, evaluar hasta dónde los maestros han sido partícipes activos y conscientes de la transformación democrática de las relaciones entre la familia, el alumnado y la escuela. Esto se necesita en tanto los actores escolares no son pizarras en blanco, sino sistemas que operan desde su propia lógica y autonomía; de ahí la imposibilidad de intentar cambios desde un paradigma impositivo. ${ }^{79}$

\section{Fuentes}

Congreso de la República de Colombia. Ley 115 de febrero 8 de 1994.

Congreso de la República. Ley 1098 de 2006 (noviembre 8).

Congreso de la República. Ley 1404 de 2010, (julio 27). Diario Oficial, año 145. No. 47783. (2010).

Congreso de la República. Ley 1620 de 2013 (marzo 20).

Congreso de Colombia. Ley No 202523 Jul 2020.

Corte Constitucional de Colombia. Sentencia t-386-1994. Límites del Reglamento educativo, http://www. corteconstitucional.gov.co/relatoria/1994/t-386-94.htm

Corte Constitucional de Colombia. Sentencia de unificación 642/98 Derecho al libre desarrollo de los niños, http://www.corteconstitucional.gov.co/ relatoria/1998/SU642-98.htm

Corte Constitucional de Colombia. Sentencia T-435/02 Derecho al debido proceso, http://www.corteconstitucional. gov.co/relatoria/2002/t-435-02.htm

Corte Constitucional de Colombia. Sentencia T-688/05 Manual de Convivencia no puede vulnerar derechos constitucionales, http://www.corteconstitucional. gov.co/RELATORIA/2005/T-688-05.htm

${ }^{79}$ Rosa Blanco, Convivencia democrática, inclusión y cultura de paz (Santiago de Chile: Unesco, 2008). 
Corte Constitucional de Colombia. Sentencia T-345/08 Derecho al libre desarrollo de la personalidad, http://www.corteconstitucional.gov.co/ relatoria/2008/T-345-08.htm

Corte Constitucional de Colombia. Sentencia T-356/13 Derecho al libre desarrollo de la personalidad, http://www.corteconstitucional.gov.co/ relatoria/2013/T-356-13.htm

Ministerio de Salud y Protección Social. Resolución 089 de 2019 (16 Ene 2019).

Presidencia de la República de Colombia. Decreto 1860 de agosto 3 de 1994.

Presidencia de la República. Decreto 1850 de agosto 13 de 2002.

Presidencia de la República. Decreto No. 1286 (27 Abr 2005).

Presidencia de la República. Decreto 1965 de 2013 (octubre 07).

\section{Referencias}

Aguilera García, Antonieta. "Desafíos conceptuales y metodológicos para evaluar la convivencia escolar". En Reflexiones sobre convivencia escolar. De las políticas públicas al salón de clases, editado por Ochoa Cervantes, Azucena y Salinas de la Vega, José Juan. Querétaro: Universidad Autónoma de Querétaro, 2018.

Asamblea Nacional Constituyente. Constitución Política de Colombia. Bogotá: Gaceta Constitucional No. 116, 1991.

Ascorra Costa, Paula, López Leiva, Verónica y Morales, Macarena. "Políticas públicas en convivencia escolar. Una visión comparada en América Latina: El caso de Chile". En Reflexiones sobre convivencia escolar. De las politicas públicas al salón de clases, editado por Ochoa Cervantes, Azucena y Salinas de la Vega, José Juan. México: Universidad Autónoma de Queretano, 2018.

Blanco, Rosa. Convivencia democrática, inclusión y cultura de paz, lecciones desde la práctica innovadora en América Latina. Santiago de Chile: Unesco, 2008.

Bocanegra Acosta, Henry y Herrera Castillo, Carlos. "La ley 1620 de 2013 y la política pública educativa de convivencia escolar en Colombia: entre 
la formalidad jurídica y la realidad social". Revista Republicana. No 23, (2017).

Cabrales Villalba, Levys Yamira, Contreras García, Nelsy, Gonzáles Romero, Luz Ángela y Rodríguez Mendoza, Yudexy. "Problemáticas de convivencia escolar en las instituciones educativas del caribe colombiano: análisis desde la pedagogía social para la cultura de paz”. Tesis de maestría en, Universidad del Norte, 2017.

Castellanos Bothia, Flor Liliana y Pérez Álvarez, Karen Estefanni. "La convivencia escolar: una arista de paz en medio del posconflicto". Temas socio-jurídicos, vol. 38. No. 76, (2019).

Castro Jiménez, Ivette Andrea. "Propuesta para la implementación de la mediación escolar como método alternativo de resolución de conflictos para la construcción de una convivencia pacifica en las instituciones educativas públicas de la ciudad de Pasto". Trabajo de especialización, Universidad de Nariño, 2013.

Cava Caballero, María Jesús. "La utilidad de la mediación como estrategia de resolución y prevención de conflictos en el ámbito escolar". Información Psicológica. No. 95, (2009).

Chaparro Caso, Alicia Aleli y Mora Osuna, Noé. "Desafíos de la evaluación de la convivencia escolar". En Reflexiones sobre convivencia escolar. De las politicas públicas al salón de clases, editado por Ochoa Cervantes, Azucena y Salinas de la Vega, José Juan. Querétaro: Universidad Autónoma de Querétaro, 2018.

Chaux, Enrique, coord. Estándares Básicos de Competencias Ciudadanas. Formar para la ciudadanía... isi es posible! Bogotá: Ministerio de Educación Nacional, 2004.

Cortés, Diego. “ABC de los Manuales de Convivencia”. Publicaciones semcucuta (2016).

Cuero, Claudia Patricia, Garcés, Sandy Leandra, Iral, Andrés Mauricio y Suárez, Adriana Patricia. "Propuesta de intervención psicopedagógica para el mejoramiento de la convivencia escolar en estudiantes de grado séptimo de cuatro instituciones públicas del oriente antioqueño". Tesis de maestría, Universidad Pontificia Bolivariana, 2018. 
Fierro-Evans, María Cecilia. "Convivencia inclusiva y democrática. Una perspectiva para gestionar la seguridad escolar". Sinéctica, revista electrónica de Educación. No. 40, (2013).

Fierro-Evans, Cecilia y Carbajal-Padilla, Patricia. "Convivencia escolar: una revisión del concepto”. Psicoperspectivas, vol. 18. No. 1, (2019).

Figueredo Ramírez, Carlos Arturo, González Santana, Jorge Eliécer, Cortázar Lemos, Héctor Julio. "Las políticas públicas educativas en Colombia y su pertinencia en los planes de desarrollo". Tesis de maestría, Universidad San Buenaventura, 2016.

Fuentes, Luz Stella y Pérez Castro, Libia María. "Convivencia escolar: una mirada desde las familias". Revista de Estudios Interdisciplinarios en Ciencias Sociales, vol. 21. No. 1, (2019).

García Escamilla, Carmen María. "Familia y Convivencia Escolar". Tesis de maestría, Universidad de Almería, 2011.

González Calderón, Martha Hilda. "Educar para la paz, una tarea de todos. Mediación escolar". Cotidiano, vol. 33. No. 208, (2018).

Guerrero Cuentas, Hilda Rosa y Cepeda, María Luz. "Uso de estrategias pedagógicas para el fortalecimiento de la convivencia escolar de jóvenes vulnerables". Revista de pedagogía, vol. 37. No. 101, (2016).

Hermosilla Benítez, Laura. "Programa de mediación escolar y formación de alumnos". Trabajo de máster, Universidad de Valladolid, 2015.

Herrera, Mauricio, Romera, Eva y Ortega Ruiz, Rosario. "Bullying y ciberbullying en Colombia; coocurrencia en adolescentes escolarizados". Revista Latinoamericana de Psicología. No. 49, (2017).

Jares, Xesús R. "El lugar del conflicto en la organización escolar". Revista Iberoamericana de Educación. No. 302, (1997).

López, Verónica, Ascorra, Paula, Litichever, Lucía y Ochoa, Azucena. "Editorial Sección Temática Violencia y Convivencia en América Latina: Políticas, prácticas, marcos de comprensión y acción". Psicoperspectivas, vol. 18. No. 1, (2019). 
Lungman, Silvia. La mediación escolar. Buenos Aires: Lugar Editorial, 1996.

Martínez, Leonel. "Análisis del sistema nacional de convivencia escolar y formación para el ejercicio de los derechos humanos, sexuales y reproductivos desde la perspectiva de la eficacia simbólica". Pensamiento jurídico. No. 49, (2018).

Ministerio de Educación, Chile. Politica nacional de convivencia escolar. Chile: Ministerio de Educación, 2019.

Ministerio de Educación de la ciudad autónoma de Buenos Aires. Ley F - $\mathrm{N}^{\circ} 3.055$ De Mediación Escolar. Buenos Aires: Ministerio de Educación, Ciencia y Tecnología.

Ministerio de Educación Nacional. Política educativa para la formación escolar en la convivencia. Bogotá: Ministerio de Educación Nacional, 2103.

Ministerio de Educación Nacional. Concepto ER-027640. Asunto: Sanciones del manual de convivencia. 2016. Bogotá: Ministerio de Educación Nacional, 2016.

Ministerio de Educación Nacional. Guía No. 1. Educación para la sexualidad y construcción de ciudadanía. Bogotá: Ministerio de Educación, 2008.

Ministerio de Educación Nacional. Guía No. 49. Guías Pedagógicas para la Convivencia Escolar. Ley 1620 de 2013 - Decreto 1965 de 2013. Bogotá: Ministerio de Educación Nacional, 2014.

Morales, Macarena y López, Verónica López. "Políticas de convivencia escolar en América Latina: cuatro perspectivas de comprensión y acción". Archivos analíticos de políticas educativas, vol. 27. No. 5 (2019).

Ortega Ruiz, Rosario y Rey, Rosario del. "La mediación escolar en el marco de la construcción de la convivencia y la prevención de la violencia". Avances en supervisión educativa: Revista de la Asociación de Inspectores de Educación de España. No. 2, (2006).

Ortega Mondaca, Raúl, Maripangui Urbina, Carolina, Ñacupil Duarte, Miguel, Cabezas Correa, Héctor y Carafi Ávalos, Eric. Orientaciones estratégicas para la formación de monitores en mediación de conflictos escolares. Santiago de Chile: Universidad de Chile, 2007. 
Peña Vera, Tania y Pirela Morillo, Johann. "La complejidad del análisis documental". Información, Cultura y Sociedad. No. 16, (2007).

Presidencia de la República, Perú. Lineamientos para la Gestión de la Convivencia Escolar, la Prevención y la Atención de la Violencia contra Niñas, Niños y Adolescentes. Lima: Ministerio de Educación, 2018.

Pulido Valero, Rosa, Fajardo, Teresa, Pleguezuelos, Laura y Gregorio, Rebeca de. "La mediación escolar en la comunidad de Madrid: Análisis del impacto de la formación en el profesorado y alumnado en el IES Las Américas de Parla". Revista de Mediación. Año 3. No. 6, (2010).

Quiñones Rivero, Edwin Davian y Valencia Quintero, Jaqueline. "Construcción de valores en la familia y convivencia escolar". Tesis de maestría, Universidad de la Sabana, 2016.

Ramírez Munar, Fanny Esperanza y Ramírez Ospina, Guiovanna. "Propuesta pedagógica con enfoque relacional para fortalecer los vínculos familiares y mejorar la convivencia". Tesis de Maestría, Universidad de la Sabana, 2016.

Rolán González, Katia, Martínez Valladares, Macarena, Parada Alfaya, Vanesa, Abilleira Escudero, Andrea y Fariña Rivera, Francisca. "Acoso escolar en primaria tras la formación en técnicas de mediación". Revista de Estudios e Investigación en Psicología y Educación. No. 2, (2017).

Ruiz Forero, José Alejandro. "Marco legal y jurisprudencial del acoso escolar en Colombia. 10 años de jurisprudencia constitucional". Revista Temas Socio Jurídicos, vol. 36. No. 71, (2016).

Salas, Karen Elena y Cómbita Niño, Harold Arturo. "Análisis de la convivencia escolar desde la perspectiva psicológica, legal y pedagógica en Colombia". Cultura, Educación y Sociedad, vol. 8. No. 2, (2017).

Tamayo, Manuel. “El análisis de las políticas públicas”. En La nueva administración pública, editado por Rafael Bañón y Ernesto Carrillo. Madrid: Alianza Editorial, 1997.

Torrego, Juan Carlos. Mediación de conflictos en instituciones educativas. Manual para la formación de formadores. Madrid: Narcea, 2000. 
Valderrama Rengifo, James. Guía No. 34. Guía para el Mejoramiento Institucional. De la Autoevaluación al Plan de Mejoramiento. Bogotá: Ministerio de Educación Nacional, 2008.

Valencia Murcia, Fernando y Mazuera, Viviana. "La figura del manual de convivencia en la vida escolar. Elementos para su comprensión". Revista cientifica Guillermo de Ockam, vol. 4. No. 1, (2006).

Vega Umbasía, Leonardo Alberto, Fernández Gallego, Álvaro Alfonso y Giraldo Navia, Néstor Raúl. "Los entornos sociales y su relación con el conflicto y la violencia escolar en las ciudades de Armenia y Manizales, Colombia". Sophia, vol. 13, No. 1, (2017).

Vizcaíno Gutiérrez, Milcíades. "La formación para la convivencia: un reto para la gestión escolar". Cuaderno venezolano de sociología, vol. 24. No. 13, (2015).

Zambrano Ramírez, Raquel. "Implantación de un programa de mediación escolar". Vía Docente. No. 1, (2015).

\section{Citar este artículo}

Benítez Moreno, Francisco Javier. "Las políticas de convivencia escolar colombianas en el contexto de América Latina". Revista Historia de la Educación Colombiana. Vol. 24 No 24 (2020): 45-75

DOI: https://doi.org/10.22267/rhec.202424.72 